\title{
Platelet-Rich Plasma (PRP) in Dental Extraction of Patients at Risk of Bisphosphonate-Related Osteonecrosis of the Jaws: A Two-Year Longitudinal Study
}

\author{
Rodolfo Mauceri ${ }^{1,2}{ }^{-}$, Vera Panzarella ${ }^{1}$, Giuseppe Pizzo ${ }^{1, *}{ }^{-}$, Giacomo Oteri ${ }^{2}{ }^{\circ}$, \\ Gabriele Cervino $^{2}$, , Giuseppina Mazzola ${ }^{3}$, Olga Di Fede ${ }^{1}$ and Giuseppina Campisi $^{1}$ (D) \\ 1 Department of Surgical, Oncological and Oral Sciences, University of Palermo, 90127 Palermo, Italy; \\ rodolfo.mauceri@unipa.it (R.M.); vera.panzarella@unipa.it (V.P.); olga.difede@unipa.it (O.D.F.); \\ giuseppina.campisi@unipa.it (G.C.) \\ 2 Department of Biomedical and Dental Sciences and Morphofunctional Imaging, University of Messina; \\ 98125 Messina, Italy; oterig@unime.it (G.O.); gabrielecervino@gmail.com (G.C.) \\ 3 Unit of Transfusion Medicine, University Hospital “Policlinico Paolo Giaccone”, 90127 Palermo, Italy; \\ giuseppina.mazzola@unipa.it \\ * Correspondence: giuseppe.pizzo@unipa.it
}

Received: 6 May 2020; Accepted: 26 June 2020; Published: 29 June 2020

\begin{abstract}
Dental extraction has often been described as the main trigger event of osteonecrosis of the jaws (ONJ). This longitudinal hospital-based study aimed to evaluate the outcome at 2 years of a standardized medical-surgical protocol for dental extraction, combined with platelet rich-plasma (PRP) application, compared with conventional protocol not combined with PRP or any other autologous platelet concentrate in cancer (ONC) and osteometabolic (OST) patients, at risk of bisphosphonate (BP)-related ONJ. Twenty patients were consecutively recruited: six received BPs for cancer skeletal-related events ( $34.17 \pm 19.97$ months), while fourteen received BPs for metabolic bone disease (74.5 \pm 34.73 months). These patients underwent a standardized protocol for dental extraction, combined with autologous PRP application in the post-extraction socket. A total of 63 dental extractions were performed (24 and 39 in ONC and OST groups, respectively). As controls, historical cases, derived from the literature and including 171 ONC and 734 OST patients, were considered. The outcome of the surgical treatment was successful in all patients treated with PRP: two years after extraction, no patient had clinical or radiological signs of ONJ. When this datum was compared with historical controls, no statistically significant differences were found $(p>0.1)$. The combination of a standardized medical-surgical protocol with PRP application may contribute to limit the occurrence of BP-related ONJ, in both ONC and OST patients. Additional prospective studies with a larger patient sample are necessary to confirm this datum.
\end{abstract}

Keywords: platelet-rich plasma; PRP; osteonecrosis of the jaws; ONJ; bisphosphonates; dental extraction

\section{Introduction}

Osteonecrosis of the jaws (ONJ) is a rare but potentially severe and debilitating complication of the jaw bones that may develop after treatments with antiresorptive or antiangiogenic drugs. It consists of progressive bone destruction in the maxillofacial area of patients exposed to ONJ-related medication, in the absence of a previous radiation treatment or metastasis $[1,2]$

Since 2003, ONJ appeared as a bisphosphonate (BP)-related class effect [3]. In the following years, ONJ cases have been reported after treatment, including other antiresorptive drugs (AR), such as denosumab, and antiangiogenic agents and other 'targeted therapy' (with and without BPs) [4-7]. 
Among the AR medication, BPs are the most common agents related to ONJ. BPs are widely prescribed to treat skeletal disorders, in order to reduce the risk of skeletal-related events, such as bone metastasis, fractures, malignant hypercalcemia and pain in cancer patients (from now on ONC), and to control the bone loss in patients affected by osteometabolic diseases (e.g., osteoporosis) (from now on OST) $[2,7]$.

Risk factors of BP-related ONJ (BRONJ) are commonly divided into three categories: systemic, drug-related and local risk factors $[2,7,8]$. ONC patients have been defined at high risk of BRONJ, even after a single dose administration of BPs, because they are contemporaneously exposed to a large number of BRONJ risk factors. OST patients, especially those in BP-treatment for a period of time greater than 3 years or shorter than 3 years and simultaneously affected by systemic or local risk factors, bear a not well defined risk of developing BRONJ, but surely lower than that in ONC patients [2,9].

BPs main function is strong inhibition of the osteoclastic activity with the suppression of bone turnover; moreover, BPs also possess anti-angiogenetic properties. The multiple and different pharmacological actions of BPs may delay or inhibit the wound healing of tooth extraction sockets, and they may be involved in BRONJ development $[2,10-13]$.

Among the local risk factors, dental extraction has been described as the main trigger event in the majority cases of reported BRONJ $[2,7,12,14-16]$. However, to date, the exact prevalence of BRONJ after tooth extraction is still not well known, and several surgical protocols for dental extraction have been proposed, with different rates of ONJ onset $[2,7,9,16,17]$.

Recently, many authors have investigated the application of autologous platelet concentrates (APC) in post-extraction sockets in patients at risk of BRONJ [18]. The application of APC was first proposed by Marx et al. in 1998; from then APC have been applied in various fields of regenerative medicine, including oral surgery $[19,20]$.

Platelet-rich plasma (PRP), belonging to APC class, is an autologous product that consists of a high concentration of platelets and a native concentration of fibrinogen [21,22]. Given the high concentration of platelets, the PRP is rich in platelets granules, which contain many substances fundamental to the promotion of the healing process (e.g., adhesive proteins, pro-coagulant factors, cytokines and chemokines), and a great number of growth factors, such as platelet-derived growth factor (PDGF), transforming growth factor-beta (TGF-b), epidermal growth factors (EGF) and vascular endothelial growth factors (VEGF) [19,21,22]. Thanks to all these components, PRP promotes angiogenesis and mucosal healing, making it an attractive product in surgical procedures on patients at risk of BRONJ $[19,21,22]$.

The aim of the present study was to evaluate the outcome at 2 years of a standardized medical-surgical protocol for dental extraction, including PRP application in post-extraction sockets, compared with conventional protocol not combined with PRP or any other APC, both in ONC and OST patients at risk of BRONJ.

\section{Materials and Methods}

\subsection{Study Design}

A longitudinal hospital-based study on patients attending the Unit of Oral Medicine of the University Hospital "Policlinico Paolo Giaccone" in Palermo (Italy) was performed. Patients were consecutively enrolled from August 2015 to April 2016. A standardized medical-surgical protocol for dental extraction [9] combined with platelet rich-plasma (PRP) application was applied to all patients enrolled. The study protocol conformed with ethical guidelines of the 1964 Declaration of Helsinki and its later amendments or comparable ethical standards, and it was approved by institutional review board of the University Hospital "Policlinico Paolo Giaccone" in Palermo (approval number 6/2015) (Table 1). Before PRP preparation and surgical procedures, all patients signed written informed consent. 
Table 1. Study steps.

\begin{tabular}{cl}
\hline Step & \\
\hline \multirow{2}{*}{ 1th } & First evaluation: \\
& - Clinical Evaluation \\
& - Computer Tomography (CT) prescription (when ONJ was suspected) \\
\hline \multirow{3}{*}{ 2nd } & Second evaluation: \\
& - Pre-operatory medical therapy prescription \\
& - Pltrasonic periodontal debridement (when required) and oral hygiene instructions \\
& Surgical procedures \\
& - Dental extraction \\
& - PRP application \\
& - Flap suture \\
\hline 4th & Suture removal and clinical control \\
\hline 5th & Follow-ups at 15 days and at one-three-six-twelve-eighteen-twenty-four months
\end{tabular}

As controls, historical cases derived from the literature were considered, differentiated between ONC $(n=171)$ and OST $(n=734)$ patients. They were discovered through published studies identified by electronic search of PubMed, Ovide/MEDLINE, Web of Science, Embase, with prespecified English language and human-studies restrictions. The literature search included clinical trials published up to 30 December 2018. During searching, the following search terms were used alone or in an alternate combination: osteonecrosis, jaw, ONJ, BRONJ, dental extraction, tooth extraction. The chosen studies reported the outcome of surgical protocols for dental extraction in ONC and OST patients at risk of BRONJ, matching for thresholds of BP exposure time, of number of teeth extracted per patient and follow-up term; patients did not receive any APC or adjuvant therapies. The studies chosen, published from 2010 to 2015, included well-defined groups of patients at risk of BRONJ (at least 6 months BP by IV exposure for ONC [23-26] and 24 months BP per os exposure for OST [23,25,27]), a well described surgical protocol (with an average of at least 2 teeth extracted for each ONC or OST patient), and a minimum follow up of 12 months. All the latter criteria, clustered for each risk group, matched those of the respective study group. Studies which did not match these criteria were not considered.

\subsection{Entry and Exclusion Criteria}

Patients were eligible for the study if they: (I) were aged $>18$ years; (II) had treatment with BPs because of the underlying disease (OST or ONC); (III) required extraction due to infective-inflammatory dental disease; (IV) had a follow-up period of at least 24 months after dental extraction; (V) had an absence, at baseline, of clinic-radiological signs of medication-related ONJ, according to the Italian Societies of Maxillo-Facial Surgery and of Oral Pathology and Medicine (SICMF and SIPMO, respectively) [28].

Patients were excluded from the study if they: (I) had clinical or radiological signs of ONJ in the surgical area; (II) had a previous history of irradiation to the head and neck area; (III) had neoplastic involvement of the jaws; (IV) were in poor general condition; (V) were pregnant or breast-feeding women.

The primary endpoint was the onset of medication-related ONJ after dental extraction.

\subsection{Clinical and Surgical Procedures}

\subsubsection{First Evaluation}

During the first visit, medical, pharmacological, and dental histories of patients were recorded; in detail. The data collected were as follows: (1) age; (2) gender; (3) reason for BPs usage; (4) BPs type; (5) duration of BPs treatment; (6) cumulative dose of BPs; (7) history of chemotherapy; (8) concurrent 
use of other AR therapy and/or anti-angiogenic medications; (9) concurrent use of steroids; (10) other concomitant diseases; (11) smoking habit.

In the presence of suspected ONJ, a computer tomography (CT) scan was requested.

\subsubsection{Second Evaluation}

All patients underwent peri-operative pharmacological treatment, based on the administration of amoxicillin/clavulanate potassium and metronidazole. Patients with a known allergy to penicillin received lincomycin. All patients signed a written informed consent form to acknowledge the off-label use of metronizadole in Italy. The use of chlorhexidine $0.2 \%$ mouthwash and sodium-hyaluronate gel (Aminogam gel, Errekappa Euroterapici S.p.A., Milan, Italy) were also prescribed (Tables 1 and 2).

Table 2. Peri-operative pharmacological treatment.

\begin{tabular}{ll}
\hline Pre- & Amoxicillin/clavulanate potassium *: 1 gr per os $3 x$ daily starting 1 day before. \\
& Metronidazole: $250 \mathrm{mg}$ per os $2 x$ daily starting 1 day before. \\
& Chlorhexidine $0.2 \%$ mouthwashes $30 \mathrm{~mL}$ swished up to $60 \mathrm{~s}, 3 x$ daily 7 days before. \\
\hline \multirow{2}{*}{ Post- } & Amoxicillin/clavulanate potassium: 1 gr per os $3 x$ daily for 7 days. \\
& Metronidazole: $250 \mathrm{mg}$ per os $2 x$ daily for 7 days. \\
& Chlorhexidine $0.2 \%$ mouthwashes $30 \mathrm{~mL}$ swished up to $60 \mathrm{~s}, 3 x$ daily 15 days post-operatively. \\
& Local application of sodium-hyaluronate $3 x$ daily 10 days post-operatively. \\
\hline
\end{tabular}

${ }^{*}$ Patients with a known allergy to penicillin received lincomycin (pre-operative regimens: $500 \mathrm{mg}$ per os $2 \mathrm{x}$ daily starting 1 day pre-operatively; post-operative regimens: $500 \mathrm{mg}$ per os $2 x$ daily for 6 days)

Autologous PRP was prepared using a commercially available system (Plateltex ACT System ${ }^{\circledR}$, Biomed, Modena, Italy), according to the manufacturer's instructions. The peripheral blood was collected in four anticoagulant citrate-dextrose, formula A (ACD-A)-containing tubes; the PRP was prepared after centrifugation at $180 \mathrm{~g}$ for $10 \mathrm{~min}$ with a common bench centrifuge. After the platelet count, the PRP was centrifuged at $1000 \mathrm{~g}$ for $10 \mathrm{~min}$ and platelet poor plasma (PPP) was removed, in order to adjust the platelet concentration to $2 \times 10^{9} / \mathrm{mL}$. One week before the surgical procedure, oral hygiene instructions were reinforced in patients with poor oral hygiene.

\subsubsection{Surgical Dental Extraction}

For all the patients, the surgical protocol included: (1) chlorhexidine $0.2 \%$ mouthwash $30 \mathrm{~mL}$ swished up to $60 \mathrm{~s}$; (2) local anesthesia, achieved using 3\% mepivacaine hydrochloride without adrenaline; (3) elevation of a full-thickness mucoperiosteal flap; (4) tooth luxation and avulsion, gently performed with elevators and forceps; (5) if necessary, subsequent osteoplasty by means of an ultrasonic surgical device (Mectron Piezosurgery Device ${ }^{\circledR}$, Mectron Medical Technology, Carasco, Italy); (6) debridement of the post-extraction socket with miller surgical curette and irrigation of the sockets with rifamycin sodium; (7) application of autologous PRP; (8) tension-free soft tissue closure (Figure 1). Before the PRP application, gelation was induced by adding the activation solution (i.e., $1 \mathrm{~mL}$ of calcium gluconate enriched batroxobin solution, from the vials provided by the manufacturer) to the PRP.

\subsubsection{Follow-Up}

The sutures were removed between 7-10 days after the surgical procedures. Follow-up visits were scheduled to confirm the healing of the sockets at 1,3, 6, 12, 18 and 24 months. Radiographic evaluations of bone healing were scheduled at 6,12 and 24 months. The successful treatment was defined as the absence of clinical and radiological signs of ONJ in the surgical area after 12 months $[28,29]$.

\subsection{Statistical Analysis and Historical Controls}

The statistical units are the patients who satisfy the inclusion criteria of the study. Descriptive statistics of all data were conventionally calculated. In bivariate analysis, the $\chi^{2}$ test or Fisher's exact 
test (if appropriate) was used to determine differences in the proportions of categorized variables. Statistical analysis was performed using Stata/SE 14.1 (Stata Corporation, College Station, TX, USA) and an alpha value of 0.05 was considered significant.
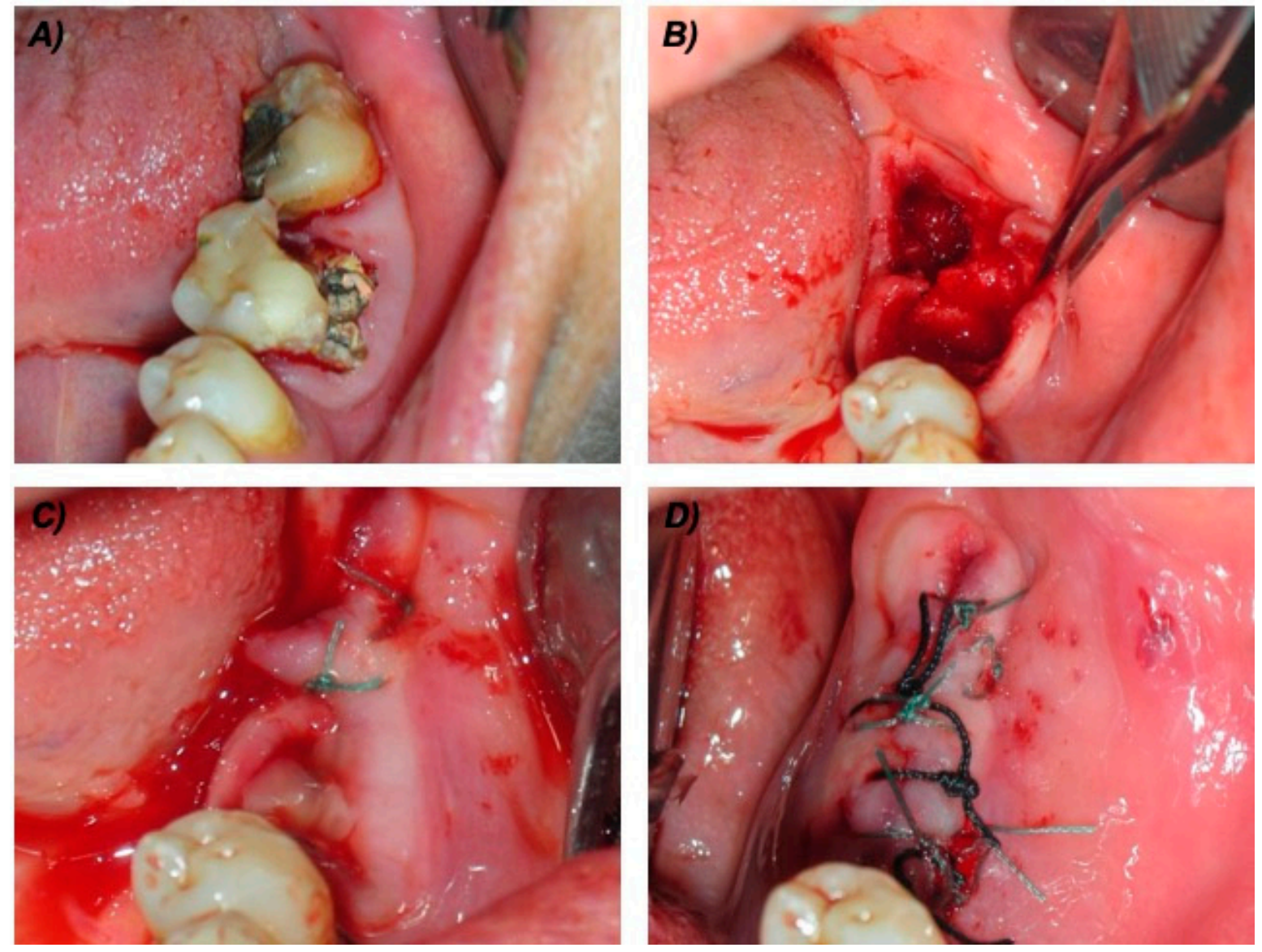

Figure 1. Surgical technical procedures in one cancer (ONC) patient taking intravenous bisphosphonates. (A) Pre-operative view of the two compromised teeth. (B) Intra-operative view after teeth extraction, bone curettage and osteoplastic procedures. (C) Packed platelet rich-plasma (PRP). (D) Sutures.

\section{Results}

During the study period, twenty patients completed the protocol. Six patients were in treatment with BPs for cancer skeletal-related events (ONC group), the other fourteen patients (70\%) were in treatment for metabolic bone disease (OST group) (Table 3).

Three patients (15\%) received concomitant systemic corticosteroid therapy, all belonging to the ONC group. Only four patients $(20 \%)$ were no longer in treatment with BPs at the moment of the surgical procedure, all belonging to the ONC group ( $7 \pm 6.5$ months).

In the OST group, the period of assumption of BPs was of $74.5 \pm 34.73$ months, most of the patients were in treatment with alendronic acid per os $(42.86 \%)$ and clodronic acid by IM $(28.57 \%)$.

Sixty-three tooth extractions were made (Figure 1a): thirty-three extractions (52.38\%) were from the maxilla (eight anterior teeth, 25 posterior teeth) and thirty $(47.62 \%)$ from the mandible (nine anterior teeth, 21 posterior teeth). Twenty-four teeth were extracted in the ONC group (four teeth/patient), while thirty-nine teeth were extracted in the OST group (2.7 teeth/patient) (Table 4). 
Table 3. Descriptive details of patients enrolled ( $n=20 ;$ ONC $=6$; OST $=14$ ).

\begin{tabular}{cc}
\hline Age (yr) & $72.35( \pm 7.19)$ \\
\hline Gender (male/female) & $4 / 16$ \\
\hline ONC patients assuming zoledronic acid & 6 \\
\hline Zoledronic acid cumulative dose $(\mathrm{mg})$ & $142( \pm 80.85)$ \\
\hline Systemic corticosteroid therapy (yes/no) & $3 / 6$ \\
\hline OST patients assuming BPs & 14 \\
\hline Alendronic acid (yes/no) & $6 / 14$ \\
\hline Alendronic acid cumulative dose $(m g)$ & $46,040( \pm 17.13)$ \\
\hline Clodronic acid (yes/no) & $4 / 14$ \\
\hline Clodronic acid cumulative dose $(m g)$ & $37,800( \pm 9.39)$ \\
\hline Ibandronic acid (yes/no) & $2 / 14$ \\
\hline Ibandronic acid cumulative dose $(m g)$ & $1 \mathrm{pt}) 5500$ \\
\hline Risedronic acid (yes/no) & $2 \mathrm{pt}) 18,000$ \\
\hline Risedronic acid cumulative dose $(\mathrm{mg})$ & $2 / 14$ \\
\hline
\end{tabular}

In detail, in the ONC group the duration of BPs therapy was of $34.17 \pm 19.97$ months; all patients were in treatment with zoledronic acid by IV, according to the standard regimen.

Table 4. Descriptive details of the extracted teeth (total $\mathrm{N}=63$ ).

\begin{tabular}{|c|c|c|c|c|}
\hline & $N$ Extraction (\%) & Maxilla/Mandible & Site & $N(\%)$ \\
\hline \multirow{4}{*}{ ONC group } & \multirow{4}{*}{$\begin{array}{c}24 \\
(38.09 \%)\end{array}$} & \multirow{2}{*}{ Maxilla } & Anterior & $3(4.8 \%)$ \\
\hline & & & Posterior & $10(15.9)$ \\
\hline & & \multirow{2}{*}{ Mandible } & Anterior & $2(3.2 \%)$ \\
\hline & & & Posterior & $9(14.3 \%)$ \\
\hline \multirow{4}{*}{ OST group } & \multirow{4}{*}{$\begin{array}{c}39 \\
(61.9 \%)\end{array}$} & \multirow{2}{*}{ Maxilla } & Anterior & $5(7.9 \%)$ \\
\hline & & & Posterior & $15(23.8 \%)$ \\
\hline & & \multirow{2}{*}{ Mandible } & Anterior & $7(11.1 \%)$ \\
\hline & & & Posterior & $12(19 \%)$ \\
\hline
\end{tabular}

Dental extraction was indicated for thirty-five teeth due to Miller degree 3 mobility (14 and 21 in the ONC and OST groups, respectively). Eighteen teeth were extracted because of large carious lesions that could not be treated conservatively (six and 12 in the ONC and OST groups, respectively). The remaining ten teeth (four and six in the ONC and OST groups, respectively) were extracted for other reasons (e.g., vertical root fracture, endodontic treatment failure).

In 24 of 63 extraction sockets (38.09\%), the healing of the oral mucosa was completed at the removal of sutures; the presence of granulation tissue was recorded in the other 39 of 63 extraction sockets $(61.9 \%)$, but, at the following visit at one month, a stable healed mucosa was recorded. In a 62-year-old woman affected by breast cancer, and in treatment with zoledronic acid for 72 months, a small area of bone exposure was detected after fifteen days, but it was resolved completely by the subsequent visit at one month, and never showed signs of ONJ in the follow up period.

At the 12-month follow-up visit, all patients $(100 \%)$ showed no clinical or radiological sign of BRONJ. At the most recent follow-up visit, all patients had intact mucosa and no clinical or radiological signs of ONJ (as example see Figures 1 and 2). 

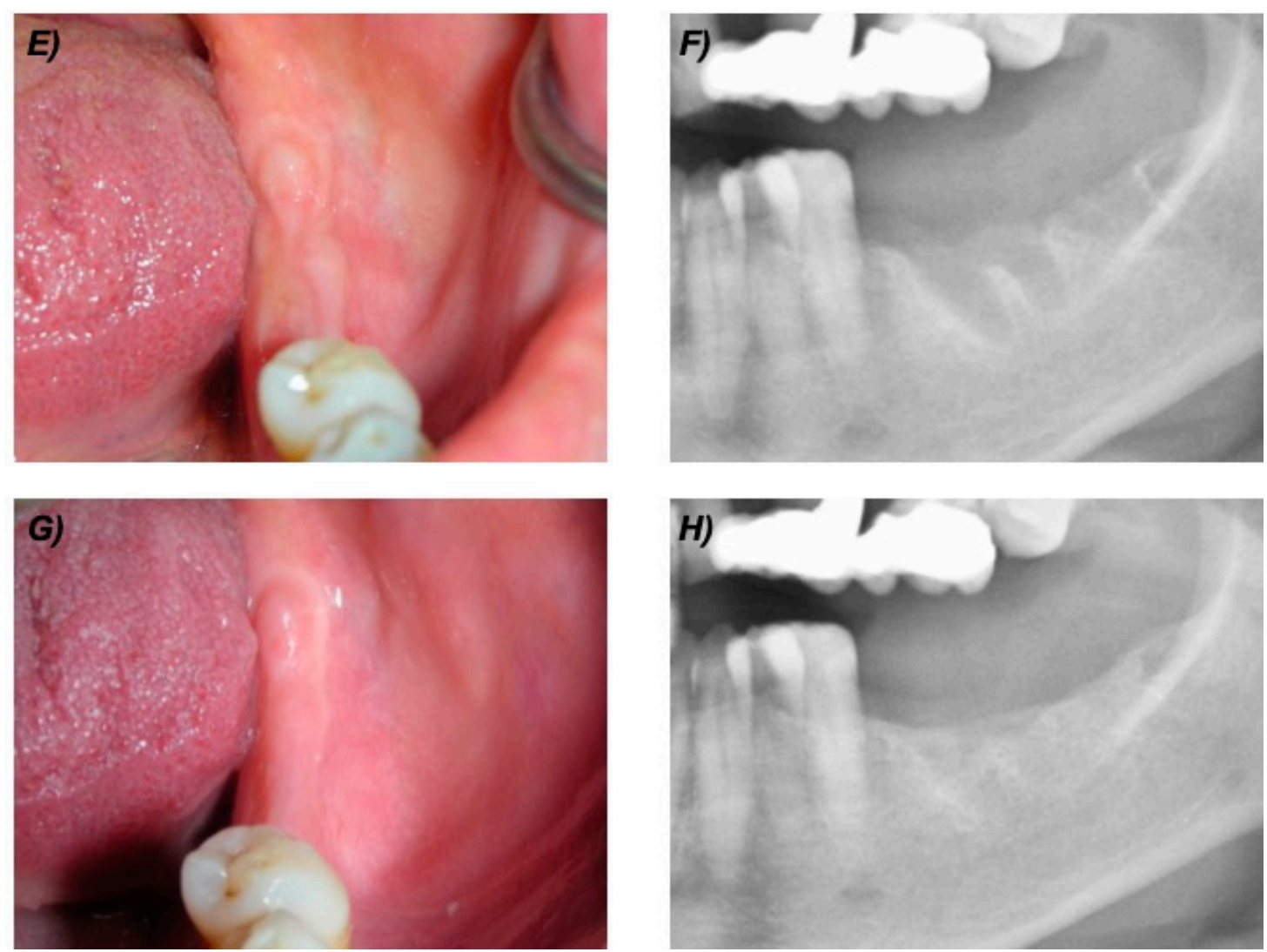

Figure 2. Clinical and radiological follow up of the same patient of Figure 1. (E) Clinical view at 6 months. (F) Detail of the panoramic X-ray at 6 months. (G) Clinical view at 24 months. (H) Detail of the panoramic $X$-ray at 24 months.

Regarding the historical controls [23-27], 16 of the 171 ONC patients developed BRONJ, while among 734 OST patients, no case of BRONJ developed. When findings from patients treated with PRP application were compared with those from selected historical controls, no statistically significant differences in the BRONJ onset were found ( $p>0.1$, for both ONC and OST patients).

\section{Discussion}

ONJ is a serious adverse reaction to certain medicines usually applied in the treatment of cancer and osteometabolic disease, related to the progressive destruction of bone in the mandible or maxilla $[2,7,9,16]$.

Great emphasis has been placed on preventive approaches to medication-related ONJ; however, for teeth with a poor prognosis, dental extraction is mandatory in order to eliminate any infective outbreaks of ONJ [9,30,31].

To date, dentoalveolar surgery is considered one of the major risk factors for developing of ONJ, with 52 to $61 \%$ of patients affected by ONJ reporting tooth extraction as the precipitating event. The estimated risk of ONJ in patients exposed to BPs for cancer therapy after tooth extraction ranges from 1.6 to $14.8 \%$, while, in osteometabolic patients, the risk of ONJ after tooth extraction is around $0.5 \%$ [7]. Recently, a growing number of authors have suggested that dental/periodontal infection prior to dental extraction, rather than surgical procedures, may represent the main local risk factor for ONJ [31-34].

Even if the pathogenesis of ONJ still remains unclear, the suppression of the osteoclastic activity and the lack of vascularization represents one of the major factors on pathogenesis of ONJ $[2,7,35]$. The application of APC may reduce the effects of BPs on post-extraction sockets, and it has been found that APC improves postsurgical wound healing in dental surgeries. The application of APC is linked to 
the great number of growth factors entrapped in it, such as PDGF, TGF-b, EGF and VEGF; the release of growth factors promotes angiogenesis and stimulates the collagen production, improving bone and mucosal healing. In addition, APC is autologous, biocompatible and a safe product [19-22].

In the present study, the application of PRP in post-extraction sockets was found to be successful in patients at risk due to BP assumption, as no occurrence of BRONJ was observed in a long term follow up period. Most post-extractive sites $(98.41 \%)$ revealed a complete healing of the oral mucosa at the removal of sutures; only one site in the ONC group showed a small area of bone exposure after fifteen days, which resolved completely before the subsequent visit, and never showed signs of ONJ throughout the follow-up period. When findings from patients treated with PRP application were compared with those from selected historical controls [23-27], no statistically significant differences in the BRONJ onset were found ( $p>0.1$, for both ONC and OST patients). Limitations of the present study are (1) the small sample size, especially for ONC group, due to the provisions of Decree of the Italian Ministry of Health of 25th November 2015 (Official Gazette of the Italian Republic no. 300 of 28th December 2015) that expects use of APC only in non-cancer patients; and (2) the historical controls. Historical cases were derived from the literature and included both ONC and OST patients who satisfy the inclusion criteria of the study (see Section 2.3).

In ONC patients, Saia et al. evaluated the occurrence of ONJ in 60 high-risk patients exposed to BPs, who collectively had 185 teeth extracted, and found that five patients in this group developed ONJ. In the whole cohort, the hazard rate of ONJ was $5.6 \%$ at 1 month $(n=4)$ and $1.5 \%$ at 6 months $(n=1)$ [23]. Ferlito et al. performed 102 tooth extractions in 43 ONC patients in treatment with zoledronate, no ONJ were observed at 12 months [24]. Sanchis-Bielsa et al. evaluated the incidence of ONJ after surgical extraction in 34 ONC patients treated with intravenous BPs. A total of 62 teeth were extracted, and eight patients developed $\mathrm{ONJ}$, the incidence of $\mathrm{ONJ}$ in terms of the number of patients was $23.5 \%$ [26] Heufelder et al. included in a prospective study 68 patients, 50 were ONC in treatment with BPs, the remaining 18 were OST patients as received oral BPs. In total, 205 teeth were removed and ONJ development was observed in three ONC patients under IV BP therapy [25]. Another two papers are worthy of note, although excluded, due to the absence of datum both on cumulative dose and months exposition from the control ONC group chosen. Mozzati et al., in a case-control study on tooth extractions in ONC patients on zoledronic acid therapy, performed 267 dental extractions in 85 patients, with five cases of ONJ development [36]; and Otto et al., in a retrospective cohort study, described the outcome of 216 dental extractions on 72 patients (43 ONC patients, 29 OST patients) [15]. Seven of the 72 patients developed ONJ during the follow-up period, three patients developed ONJ $(4.2 \%)$ in four of the 216 extraction sites (1.9\%). Regarding the occurrence of wound healing disturbance and ONJ development, a malignant underlying disease and an intravenous route of BPs administration was more common (85.7\%) [15]. In OST patients, Mozzati et al. described no evidence of ONJ on 700 patients and 1480 extractions [27]. Jeong et al. investigated the incidence of ONJ patients after surgical tooth extractions (651 teeth) in OST patients exposed to oral BPs (320 patients). Indeed, 11 patients developed ONJ with an uncommonly high incidence rate (3.44\%) [37]. These cases, however, were not included in the statistical analysis, due to the short follow-up period.

Finally, it should be noted that the present study has a long follow-up period (24 months), that is twice as long as the period suggested to exclude the onset of ONJ, following surgical procedures on patients at risk $[28,38,39]$. This characteristic of the study design counterbalances the aforementioned limitations.

Due to their properties, APC have been successfully applied in the prevention of BRONJ by several authors; no studies, however, evaluated the application of PRP in post-extraction dental socket $[18,36,40-45]$. Scoletta et al. described the application of plasma rich in growth factors (PRGF) in a protocol for tooth extraction in ONC patients treated with intravenous BPs in two different studies with a follow up of at least 12 months. In the first report, sixty-four patients in treatment with intravenous BPs (60 ONC pts, four OST patients) were enrolled; five ONC patients developed ONJ in post-extraction sites (2.27\%) [44]. In the second study sixty-three patients were enrolled (57 ONC pts, 
six OST pts), with one ONJ (0.99\%) in an ONC patient [45]. Mozzati et al. described a case-control study of tooth extraction on 176 ONC patients treated with intravenous BPs. In the study group (91 ONC pts, 542 extractions), the application of PRGF in tooth extraction was successful, with no evidence of ONJ with a follow up period of 24 to 60 months. Five ONJ lesions were evidenced in the control group (85 pts, 267 extractions) [36]. In a systematic review of the literature, Del Fabbro evaluated these three prospective studies, defining the success rate of $99.05 \%$ on a tooth basis and $97.26 \%$ on a patient basis [18]. Recently, Asaka et al. described a case-control study, in which the application of platelet rich-fibrin (PRF) dental extraction procedures on 102 OST patients was evaluated. No BRONJ were observed in the study group ( 29 pts, 52 extractions); while, nine patients in the control group (73 pts, 166 extractions) showed a delayed recovery with bone exposure 4 weeks after surgical procedures. The authors reported wound closure within 8 weeks and no evidence of ONJ; however, the follow up period of the study was relatively short (i.e., 3 months) [43].

\section{Conclusions}

Although several protocols are available, well defined guidelines to safely manage mandatory dental extractions are still missing. PRP is an autologous product, biocompatible, and rich in growth factors that seem to enhance the healing of tissues in post-surgical wounds. The proposed standardized medical-surgical protocol, based on professional oral hygiene, chlorhexidine mouthwash, perioperative antibiotic prophylaxis, atraumatic surgery and first intention wound closure, combined with PRP application in post-extraction socket, may contribute to limit the occurrence of BP-related ONJ, both in ONC and OST patients. Additional prospective studies with a larger patient sample are necessary to confirm this datum.

Author Contributions: Conceptualization, R.M. and O.D.F.; methodology, G.C. (Giuseppina Campisi); validation, G.M.; investigation, R.M., V.P. and G.M.; writing-original draft preparation, R.M.; writing-review and editing, G.P. and G.O.; visualization, G.C. (Gabriele Cervino); supervision, G.C. (Giuseppina Campisi); project administration, G.C. (Giuseppina Campisi). All authors have read and agreed to the published version of the manuscript.

Funding: This research received no external funding.

Acknowledgments: The assistance provided during the course of the research by Antonino Albanese, former PhD student, was greatly appreciated.

Conflicts of Interest: The authors declare no conflict of interest.

\section{References}

1. Bedogni, A.; Campisi, G.; Fusco, V. Medication Related Osteonecrosis of the Jaw (MRONJ); Qeios: London, UK, 2018.

2. Campisi, G.; Fedele, S.; Fusco, V.; Pizzo, G.; Di Fede, O.; Bedogni, A. Epidemiology, clinical manifestations, risk reduction and treatment strategies of jaw osteonecrosis in cancer patients exposed to antiresorptive agents. Future Oncol. 2014, 10, 257-275. [CrossRef] [PubMed]

3. Marx, R.E. Pamidronate (Aredia) and zoledronate (Zometa) induced avascular necrosis of the jaws: A growing epidemic. J. Oral Maxillofac. Surg. 2003, 61, 1115-1117. [CrossRef]

4. Fusco, V.; Santini, D.; Armento, G.; Tonini, G.; Campisi, G. Osteonecrosis of jaw beyond antiresorptive (bone-targeted) agents: New horizons in oncology. Expert Opin. Drug Saf. 2016, 15, 925-935. [CrossRef]

5. Mauceri, R.; Panzarella, V.; Morreale, I.; Campisi, G. Medication-related osteonecrosis of the jaw in a cancer patient receiving lenvatinib. Int. J. Oral Maxillofac. Surg. 2019, 48, 1530-1532. [CrossRef]

6. Nicolatou-Galitis, O.; Kouri, M.; Papadopoulou, E.; Vardas, E.; Galiti, D.; Epstein, J.B.; Elad, S.; Campisi, G.; Tsoukalas, N.; Bektas-Kayhan, K.; et al. Osteonecrosis of the jaw related to non-antiresorptive medications: A systematic review. Support. Care Cancer 2019, 27, 383-394. [CrossRef]

7. Ruggiero, S.L.; Dodson, T.B.; Fantasia, J.; Goodday, R.; Aghaloo, T.; Mehrotra, B.; O’Ryan, F. American association of oral and maxillofacial surgeons position paper on medication-related osteonecrosis of the jaw-2014 update. J. Oral Maxillofac. Surg. 2014, 72, 1938-1956. [CrossRef] [PubMed] 
8. Nisi, M.; La Ferla, F.; Karapetsa, D.; Gennai, S.; Miccoli, M.; Baggiani, A.; Graziani, F.; Gabriele, M. Risk factors influencing BRONJ staging in patients receiving intravenous bisphosphonates: A multivariate analysis. Int. J. Oral Maxillofac. Surg. 2015, 44, 586-591. [CrossRef]

9. Di Fede, O.; Panzarella, V.; Mauceri, R.; Fusco, V.; Bedogni, A.; Lo Muzio, L.; Board, S.O.; Campisi, G. The dental management of patients at risk of medication-related osteonecrosis of the jaw: New paradigm of primary prevention. BioMed Res. Int. 2018, 2018, 2684924. [CrossRef] [PubMed]

10. Kobayashi, Y.; Hiraga, T.; Ueda, A.; Wang, L.; Matsumoto-Nakano, M.; Hata, K.; Yatani, H.; Yoneda, T. Zoledronic acid delays wound healing of the tooth extraction socket, inhibits oral epithelial cell migration, and promotes proliferation and adhesion to hydroxyapatite of oral bacteria, without causing osteonecrosis of the jaw, in mice. J. Bone Miner. Metab. 2010, 28, 165-175. [CrossRef]

11. Milstein, D.M.J.; Lindeboom, J.A.H.; Ince, C. The influence of zoledronic acid and cyclophosphamide on microcirculation regeneration in healing oral mucosal flaps. Arch. Oral Biol. 2011, 56, 599-606. [CrossRef]

12. Otto, S.; Schreyer, C.; Hafner, S.; Mast, G.; Ehrenfeld, M.; Stürzenbaum, S.; Pautke, C. Bisphosphonate-related osteonecrosis of the jaws-Characteristics, risk factors, clinical features, localization and impact on oncological treatment. J. Cranio Maxillofac. Surg. 2012, 40, 303-309. [CrossRef]

13. Fung, P.L.; Nicoletti, P.; Shen, Y.; Porter, S.; Fedele, S. Pharmacogenetics of Bisphosphonate-associated Osteonecrosis of the Jaw. Oral Maxillofac. Surg. Clin. N. Am. 2015, 27, 537-546. [CrossRef]

14. Bodem, J.P.; Kargus, S.; Eckstein, S.; Saure, D.; Engel, M.; Hoffmann, J.; Freudlsperger, C. Incidence of bisphosphonate-related osteonecrosis of the jaw in high-risk patients undergoing surgical tooth extraction. J. Cranio Maxillofac. Surg. 2015, 43, 510-514. [CrossRef]

15. Otto, S.; Troltzsch, M.; Jambrovic, V.; Panya, S.; Probst, F.; Ristow, O.; Ehrenfeld, M.; Pautke, C. Tooth extraction in patients receiving oral or intravenous bisphosphonate administration: A trigger for BRONJ development? J. Cranio Maxillofac. Surg. 2015, 43, 847-854. [CrossRef] [PubMed]

16. Beth-Tasdogan, N.H.; Mayer, B.; Hussein, H.; Zolk, O. Interventions for managing medication-related osteonecrosis of the jaw. Cochrane Database Syst. Rev. 2017, 10, CD012432. [CrossRef]

17. Diniz-Freitas, M.; Limeres, J. Prevention of medication-related osteonecrosis of the jaws secondary to tooth extractions. A systematic review. Med. Oral Patol. Oral Cir. Bucal 2016, 21, e250-e259. [CrossRef]

18. Del Fabbro, M.; Gallesio, G.; Mozzati, M. Autologous platelet concentrates for bisphosphonate-related osteonecrosis of the jaw treatment and prevention. A systematic review of the literature. Eur. J. Cancer 2015, 51, 62-74. [CrossRef]

19. Albanese, A.; Licata, M.E.; Polizzi, B.; Campisi, G. Platelet-rich plasma (PRP) in dental and oral surgery: From the wound healing to bone regeneration. Immun. Ageing 2013, 10, 23. [CrossRef]

20. Del Fabbro, M.; Bucchi, C.; Lolato, A.; Corbella, S.; Testori, T.; Taschieri, S. Healing of Postextraction Sockets Preserved With Autologous Platelet Concentrates. A Systematic Review and Meta-Analysis. J. Oral Maxillofac. Surg. 2017, 75, 1601-1615. [CrossRef]

21. Dohan Ehrenfest, D.M.; Andia, I.; Zumstein, M.A.; Zhang, C.-Q.; Pinto, N.R.; Bielecki, T. Classification of platelet concentrates (Platelet-Rich Plasma-PRP, Platelet-Rich Fibrin-PRF) for topical and infiltrative use in orthopedic and sports medicine: Current consensus, clinical implications and perspectives. Muscles. Ligaments Tendons J. 2014, 4, 3-9. [CrossRef]

22. Del Corso, M.; Vervelle, A.; Simonpieri, A.; Jimbo, R.; Inchingolo, F.; Sammartino, G.; Dohan Ehrenfest, D.M. Current knowledge and perspectives for the use of platelet-rich plasma (PRP) and platelet-rich fibrin (PRF) in oral and maxillofacial surgery part 1: Periodontal and dentoalveolar surgery. Curr. Pharm. Biotechnol. 2012, 13, 1207-1230. [CrossRef] [PubMed]

23. Saia, G.; Blandamura, S.; Bettini, G.; Tronchet, A.; Totola, A.; Bedogni, G.; Ferronato, G.; Nocini, P.F.; Bedogni, A. Occurrence of Bisphosphonate-Related Osteonecrosis of the Jaw After Surgical Tooth Extraction. J. Oral Maxillofac. Surg. 2010, 68, 797-804. [CrossRef] [PubMed]

24. Ferlito, S.; Puzzo, S.; Liardo, C. Preventive protocol for tooth extractions in patients treated with zoledronate: A case series. J. Oral Maxillofac. Surg. 2011,69, e1. [CrossRef]

25. Heufelder, M.J.; Hendricks, J.; Remmerbach, T.; Frerich, B.; Hemprich, A.; Wilde, F. Principles of oral surgery for prevention of bisphosphonate-related osteonecrosis of the jaw. Oral Surg. Oral Med. Oral Pathol. Oral Radiol. 2014, 117, e429-e435. [CrossRef] [PubMed] 
26. Sanchis-Bielsa, J.M.; Bagán, J.V.; Murillo, J.; Díaz, J.M.; Asensio, L. Risk of developing BRONJ among patients exposed to intravenous bisphosphonates following tooth extraction. Quintessence Int. (Berlin) 2014, 45, 769-777. [CrossRef]

27. Mozzati, M.; Arata, V.; Gallesio, G. Tooth extraction in osteoporotic patients taking oral bisphosphonates. Osteoporos. Int. 2013, 24, 1707-1712. [CrossRef]

28. Bedogni, A.; Campisi, G.; Fusco, V.; Agrillo, A. Raccomandazioni Clinico-Terapeutiche Sull'osteonecrosi Delle Ossa Mascellari Associata A Bisfosfonati E Sua Prevenzione; Cleup: Padova, Italy, 2013.

29. Bedogni, A.; Fusco, V.; Agrillo, A.; Campisi, G. Learning from experience. Proposal of a refined definition and staging system for bisphosphonate-related osteonecrosis of the jaw (BRONJ). Oral Dis. 2012, 18, 621-623. [CrossRef] [PubMed]

30. Yarom, N.; Shapiro, C.L.; Peterson, D.E.; Van Poznak, C.H.; Bohlke, K.; Ruggiero, S.L.; Migliorati, C.A.; Khan, A.; Morrison, A.; Anderson, H.; et al. Medication-related osteonecrosis of the jaw: MASCC/ISOO/ASCO clinical practice guideline. J. Clin. Oncol. 2019, 37, 2270-2290. [CrossRef]

31. Nicolatou-Galitis, O.; Papadopoulou, E.; Vardas, E.; Kouri, M.; Galiti, D.; Galitis, E.; Alexiou, K.; Tsiklakis, K.; Ardavanis, A.; Razis, E.; et al. Alveolar bone histological necrosis observed prior to extractions in patients, who received bone targeting agents. Oral Dis. 2020. [CrossRef]

32. Nicolatou-Galitis, O.; Razis, E.; Galiti, D.; Galitis, E.; Labropoulos, S.; Tsimpidakis, A.; Sgouros, J.; Karampeazis, A.; Migliorati, C. Periodontal disease preceding osteonecrosis of the jaw (ONJ) in cancer patients receiving antiresorptives alone or combined with targeted therapies: Report of 5 cases and literature review. Oral Surg. Oral Med. Oral Pathol. Oral Radiol. 2015, 120, 699-706. [CrossRef] [PubMed]

33. Soutome, S.; Hayashida, S.; Funahara, M.; Sakamoto, Y.; Kojima, Y.; Yanamoto, S.; Umeda, M. Factors affecting development of medication-related osteonecrosis of the jaw in cancer patients receiving high-dose bisphosphonate or denosumab therapy: Is tooth extraction a risk factor? PLoS ONE 2018, 13. [CrossRef]

34. Hasegawa, T.; Hayashida, S.; Kondo, E.; Takeda, Y.; Miyamoto, H.; Kawaoka, Y.; Ueda, N.; Iwata, E.; Nakahara, H.; Kobayashi, M.; et al. Medication-related osteonecrosis of the jaw afte tooth extraction in cancer patients: A multicenter retrospective study. Osteoporos. Int. 2019, 30, 231-239. [CrossRef] [PubMed]

35. George, E.L.; Lin, Y.-L.; Saunders, M.M. Bisphosphonate-related osteonecrosis of the jaw: A mechanobiology perspective. Bone Rep. 2018, 8, 104-109. [CrossRef] [PubMed]

36. Mozzati, M.; Arata, V.; Gallesio, G. Tooth extraction in patients on zoledronic acid therapy. Oral Oncol. 2012, 48, 817-821. [CrossRef] [PubMed]

37. Jeong, H.-G.; Hwang, J.J.; Lee, J.-H.; Kim, Y.H.; Na, J.Y.; Han, S.-S. Risk factors of osteonecrosis of the jaw after tooth extraction in osteoporotic patients on oral bisphosphonates. Imaging Sci. Dent. 2017, 47, 45-50. [CrossRef]

38. Bedogni, A.; Saia, G.; Bettini, G.; Tronchet, A.; Totola, A.; Bedogni, G.; Ferronato, G.; Nocini, P.F.; Blandamura, S. Long-term outcomes of surgical resection of the jaws in cancer patients with bisphosphonate-related osteonecrosis. Oral Oncol. 2011, 47, 420-424. [CrossRef]

39. Kyrgidis, A.; Koloutsos, G.; Vahtsevanos, K. Treatment protocols of bisphosphonate-related osteonecrosis of the jaws. Head Neck 2009, 1112-1113. [CrossRef]

40. Mozzati, M.; Gallesio, G.; Arata, V.; Pol, R.; Scoletta, M. Platelet-rich therapies in the treatment of intravenous bisphosphonate-related osteonecrosis of the jaw: A report of 32 cases. Oral Oncol. 2012, 48, 469-474. [CrossRef]

41. Longo, F.; Guida, A.; Aversa, C.; Pavone, E.; Di Costanzo, G.; Ramaglia, L.; Ionna, F.; Di Costanzo, G.; Ramaglia, L.; Ionna, F. Platelet Rich Plasma in the Treatment of Bisphosphonate-Related Osteonecrosis of the Jaw: Personal Experience and Review of the Literature. Int. J. Dent. 2014, 2014, 298945. [CrossRef]

42. Mauceri, R.; Panzarella, V.; Maniscalco, L.; Bedogni, A.; Licata, M.E.; Albanese, A.; Toia, F.; Cumbo, E.M.G.; Mazzola, G.; Di Fede, O.; et al. Conservative Surgical Treatment of Bisphosphonate-Related Osteonecrosis of the Jaw with Er, Cr: YSGG Laser and Platelet-Rich Plasma: A Longitudinal Study. BioMed Res. Int. 2018, 2018. [CrossRef]

43. Asaka, T.; Ohga, N.; Yamazaki, Y.; Sato, J.; Satoh, C.; Kitagawa, Y. Platelet-rich fibrin may reduce the risk of delayed recovery in tooth-extracted patients undergoing oral bisphosphonate therapy: A trial study. Clin. Oral Investig. 2017, 21, 2165-2172. [CrossRef] [PubMed] 
44. Scoletta, M.; Arduino, P.G.; Pol, R.; Arata, V.; Silvestri, S.; Chiecchio, A.; Mozzati, M. Initial experience on the outcome of teeth extractions in intravenous bisphosphonate-treated patients: A cautionary report. J. Oral Maxillofac. Surg. 2011, 69, 456-462. [CrossRef] [PubMed]

45. Scoletta, M.; Arata, V.; Arduino, P.G.; Lerda, E.; Chiecchio, A.; Gallesio, G.; Scully, C.; Mozzati, M. Tooth extractions in intravenous bisphosphonate-treated patients: A refined protocol. J. Oral Maxillofac. Surg. 2013, 71, 994-999. [CrossRef] [PubMed]

C 2020 by the authors. Licensee MDPI, Basel, Switzerland. This article is an open access article distributed under the terms and conditions of the Creative Commons Attribution (CC BY) license (http://creativecommons.org/licenses/by/4.0/). 